\title{
Mechanisms underlying the attenuation of chronic inflammatory diseases by aged garlic extract: Involvement of the activation of AMP-activated protein kinase (Review)
}

\author{
SATOMI MIKI ${ }^{1}$, JUN-ICHIRO SUZUKI ${ }^{1}$, KAYO KUNIMURA $^{1}$ and NAOAKI MORIHARA ${ }^{1,2}$ \\ ${ }^{1}$ Central Research Institute, Wakunaga Pharmaceutical Co., Ltd., Akitakata-shi, Hiroshima 739-1195, Japan; \\ ${ }^{2}$ Research and Development, Wakunaga of America Co., Ltd., Mission Viejo, CA 92691, USA
}

Received September 4, 2019; Accepted October 9, 2019

DOI: $10.3892 /$ etm.2019.8372

\begin{abstract}
AMP-activated protein kinase (AMPK) is an ubiquitously expressed serine/threonine kinase and an important regulator of energy metabolism. The decreased activity of AMPK induced by low-grade chronic inflammation has been implicated in several diseases, including type 2 diabetes and atherosclerosis. However, the activation of AMPK by natural and synthetic products can ameliorate these diseases through the inhibition of inflammation. For example, aged garlic extract (AGE) has been shown to enhance the phosphorylation of Thr172 of the $\alpha$-subunit of AMPK in several tissues of disease model animals. In addition, AGE has been reported to suppress the progression of atherosclerotic plaque formation in an animal model of atherosclerosis. Moreover, AGE has been found to decrease the level of plasma glycated albumin and to improve hyperglycemia in an animal model of type 2 diabetes.
\end{abstract}

Correspondence to: Ms. Satomi Miki, Central Research Institute, Wakunaga Pharmaceutical Co., Ltd., 1624 Shimokotachi, Koda-cho, Akitakata-shi, Hiroshima 739-1195, Japan

E-mail: miki_s@wakunaga.co.jp

Abbreviations: ABCA1, ATP-binding cassette protein A1; AGE, aged garlic extract; AMP, adenosine monophosphate; AMPK, AMP-activated protein kinase; ApoE-KO mice, apolipoprotein E-knockout mice; ATP, adenosine triphosphate; CaMKK $\beta$, calcium/ calmodulin-dependent protein kinase kinase $\beta$; CCL2, C-C motif chemokine 2; CRP, C-reactive protein; DAMPs, damage-associated molecular patterns; FAS, fatty acid synthase; FFA, free fatty acid; GLUT4, glucose transporter 4; IL, interleukin; IRAK4, interleukin-1 receptor-associated kinase 4; LDLR, low-density lipoprotein receptor; LKB1, liver kinase $\mathrm{B} 1$; $\mathrm{MCP}-1$, monocyte chemoattractant protein-1; MyD88, myeloid differentiation primary response 88; PPRs, pattern-recognition receptors; SAC, $S$-allylcysteine; SAMC, S-allylmercaptocysteine; SREBP-1c, sterol regulatory element-binding protein-1c; S1PC, S-1-propenylcysteine; TLR, Toll-like receptor; TNF- $\alpha$, tumor necrosis factor- $\alpha$; TSOD mice, Tsumura Suzuki Obese Diabetes mice

Key words: aged garlic extract, AMP-activated protein kinase, chronic inflammatory diseases, inflammation, Toll-like receptor signaling pathway
These inhibitory effects of AGE are induced by the suppression of the inflammatory response. In the present review, we discuss the mechanisms through which AGE activates AMPK, as well as the mechanisms through which the activation of AMPK by AGE modulates the inflammatory response in disease models.

\section{Contents}

1. Introduction

2. Induction of AMPK phosphorylation by AGE and its constituents

3. Inhibition of plaque formation and induction of AMPK activation by AGE

4. Suppression of plasma glycated albumin level and induction of AMPK activation by AGE

5. Conclusions

\section{Introduction}

AMP-activated protein kinase (AMPK) is a master regulator of energy metabolism and forms a heterotrimer consisting of a catalytic $\alpha$-subunit and two regulatory subunits ( $\beta$ and $\gamma$ ). AMPK is activated via the phosphorylation at Thr172 of the $\alpha$-subunit by liver kinase B1 (LKB1) through the interaction of adenosine monophosphate (AMP) with the AMPK $\gamma$-subunit under nutrient starvation (1-3). The other mechanism of AMPK activation is the calcium-dependent phosphorylation at Thr172 of the $\alpha$ subunit by calcium/calmodulin-dependent protein kinase kinase $\beta$ (CaMKK $\beta)(2,3)$. AMPK contributes to several cellular events, such as protein synthesis, lipid metabolism, glucose metabolism, anti-inflammation, redox regulation and anti-aging (1-3). In addition, AICAR, an AMPK activator, has been shown to suppress several diseases, such as acute and relapsing colitis (4), autoimmune encephalomyelitis (5) and acute lung injury (6). Therefore, the activation of AMPK may ameliorate inflammatory diseases.

Chronic inflammation has been reported to be a key event in the development and progression of several diseases (7-10) and 
is triggered by multiple immune cells, including macrophages, T lymphocytes and mast cells (11). Damage-associated molecular patterns (DAMPs), such as proteins, peptides, fatty acids and lipoproteins derived from dead cells are recognized by pattern-recognition receptors (PRRs) expressed on the immune cell surface and induce the secretion of inflammatory molecules, including tumor necrosis factor- $\alpha$ (TNF- $\alpha$ ), $\mathrm{C}-\mathrm{C}$ motif chemokine 2 /monocyte chemoattractant protein-1 (CCL2/MCP-1) and C-reactive protein (CRP) (12-14). These molecules damage various tissues and induce chronic inflammatory diseases, such as atherosclerosis and type 2 diabetes.

Aged garlic extract (AGE) is produced by aging garlic (Allium sativum L.) in a water-ethanol mixture for $>10$ months at room temperature and contains several characteristic sulfur compounds, such as $S$-allylcysteine (SAC), $S$-1-propenylcysteine (S1PC) and $S$-allylmercaptocysteine (SAMC) $(15,16)$. AGE has been shown to exert immunomodulatory effects (17-20), anti-atherosclerotic effects (21-25), anti-hypertensive effects (26-29), antioxidant effects (30-32) and blood flow-improving effects (33) in clinical and animal studies. In addition, recent studies have suggested that AGE and its components can induce the activation of AMPK in several cell types and tissues (19,23,34-37).

In the present review, we summarize the AMPK-activating effects of AGE and its components, and discuss the association between AMPK activation and the ameliorating effects of AGE on several inflammatory diseases.

\section{Induction of AMPK phosphorylation by AGE and its constituents}

AGE has been shown to trigger the activation of AMPK in the liver, adipose tissues and gastrocnemius muscles in a model of type 2 diabetes (34). In addition, AGE has been shown to induce the phosphorylation of AMPK in the liver in a mouse model of atherosclerosis (23). Accordingly, researchers have demonstrated that AGE enhances AMPK activity in various tissues and animal models. However, the mechanisms underlying AGE-induced AMPK activation are not yet fully understood. Several natural compounds have been shown to activate AMPK. Resveratrol from red grapes, curcumin from the turmeric, and berberine from Coptis chinensis trigger the phosphorylation of Thr172 of the AMPK $\alpha$-subunit by increasing the AMP/ATP ratio $(38,39)$. These compounds decrease ATP production through various mechanisms. Resveratrol and curcumin inhibit the production of ATP by suppressing mitochondrial F1F0-ATPase/ATP synthase, whereas berberine decreases ATP production by blocking respiratory chain complex I $(38,39)$. SAMC has been shown to increase the phosphorylation of LKB1 in the liver (37), whereas SAC promotes the activation of $\mathrm{CaMKK} \beta$ in human hepatocyte HepG2 cells (35). Therefore, both SAMC and SAC are major constituents of $\operatorname{AGE}(15,16)$, and they are involved in the induction of the phosphorylation of AMPK by increasing the AMP/ATP ratio and/or intracellular $\mathrm{Ca}^{2+}$ concentration. Consequently, AGE containing these components may induce the phosphorylation of AMPK through these pathways.

\section{Inhibition of plaque formation and induction of AMPK activation by AGE}

Atherosclerosis is a vascular inflammatory disease, which causes macrophage infiltration, atherosclerotic lesion formation and defective efferocytosis (40). AMPK regulates cell apoptosis, inflammation, cholesterol efflux and efferocytosis via autophagy; however, its function is impaired in atherosclerosis (41-44). AGE has been shown to enhance the phosphorylation of AMPK in the livers of apolipoprotein $\mathrm{E}$ knockout (ApoE-KO) mice, a mouse model of atherosclerosis (23). It has previously been suggested that the activation of AMPK improves several processes in atherosclerosis. Li et al reported that the administration of the AMPK activator, S17834, reduced lipogenesis and the atherosclerotic plaque region by inhibiting sterol regulatory element-binding protein 1c (SREBP-1c) cleavage and nuclear translocation in low-density lipoprotein receptor (LDLR)-knockout mice (45). In addition, xanthohumol derived from the hop plant lowers plasma lipids and inflammatory chemokine production by activating AMPK in the liver (46). Furthermore, berberine has been shown to suppress plaque formation by inhibiting oxidative stress and vascular inflammation through the activation of the AMPK-nuclear respiratory factor 1 signaling pathway (47). These findings suggest that AMPK inhibits plaque formation by modulating lipid metabolism, as well as by exerting antioxidant and anti-inflammatory effects.

AGE has been shown to suppress lipid deposition in the aorta and to prevent the elevation of serum CRP levels in ApoE-KO mice $(22,23)$. CRP secreted from hepatocytes is a marker of chronic inflammation and systemic inflammation induced by pro-inflammatory cytokines $(48,49)$. PRRs, including TLRs trigger the production of pro-inflammatory cytokines by recognizing not only foreign pathogens, but also cellular components, such as proteins, peptides, fatty acid and lipoprotein, that are derived from dead cells. Accordingly, chronic inflammation is caused by the continuous stimulation of these components (12-14). AGE has been shown to inhibit the TLR signaling pathway by reducing the phosphorylation of interleukin-1 receptor-associated kinase 4 (IRAK4), which is a key regulator of TLR signaling, in ApoE-KO mice (23). The activation of AMPK also contributes to the inhibition of the TLR signaling pathway via several mechanisms $(6,50,51)$. $\mathrm{S} 1 \mathrm{PC}$ induces the degradation of myeloid differentiation primary response 88 (MyD88) by activating AMPK-mediated autophagy (19). SAC increases cholesterol efflux by the induction of ATP-binding cassette protein A1 (ABCA1) expression in THP-1 cells (52). In addition, the pharmacological or genetic activation of AMPK increases ABCA1 expression through transcriptional activator liver $\mathrm{X}$ receptor $\alpha$, and promotes cholesterol efflux (53). Therefore, the activation of AMPK is an important event for the amelioration of atherosclerosis and may be a therapeutic target for this disease (Fig. 1).

\section{Suppression of plasma glycated albumin level and induction of AMPK activation by AGE}

Type 2 diabetes is characterized by hyperglycemia and dyslipidemia associated with insulin resistance (54-56). In addition, inflammation contributes to the disruption of insulin 


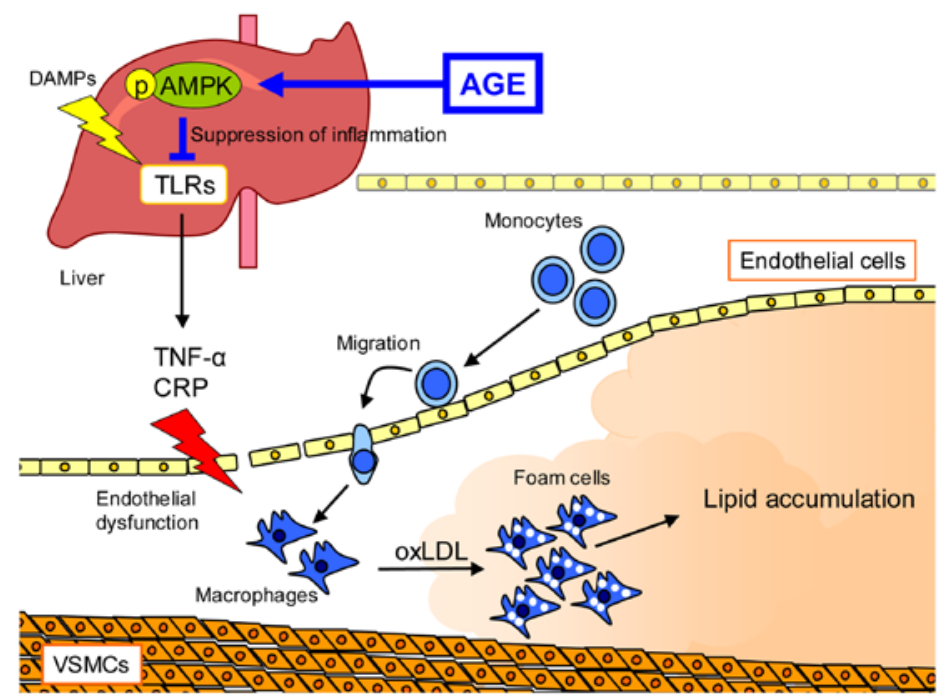

Figure 1. Effect of AGE on plaque formation. AGE inhibits the TLR signaling pathway by activating AMPK and consequently suppresses the production of TNF- $\alpha$ and CRP in the liver. The reduction of pro-inflammatory cytokines decreases the infiltration of immune cells into the artery wall, which in turn suppresses plaque formation. AGE, aged garlic extract; AMPK, AMP-activated protein kinase; CRP, C-reactive protein; DAMPs, damage-associated molecular patterns; oxLDL, oxidized low-density lipoprotein; TLRs, Toll-like receptors; TNF- $\alpha$, tumor necrosis factor- $\alpha$; VSMCs, vascular smooth muscle cells.

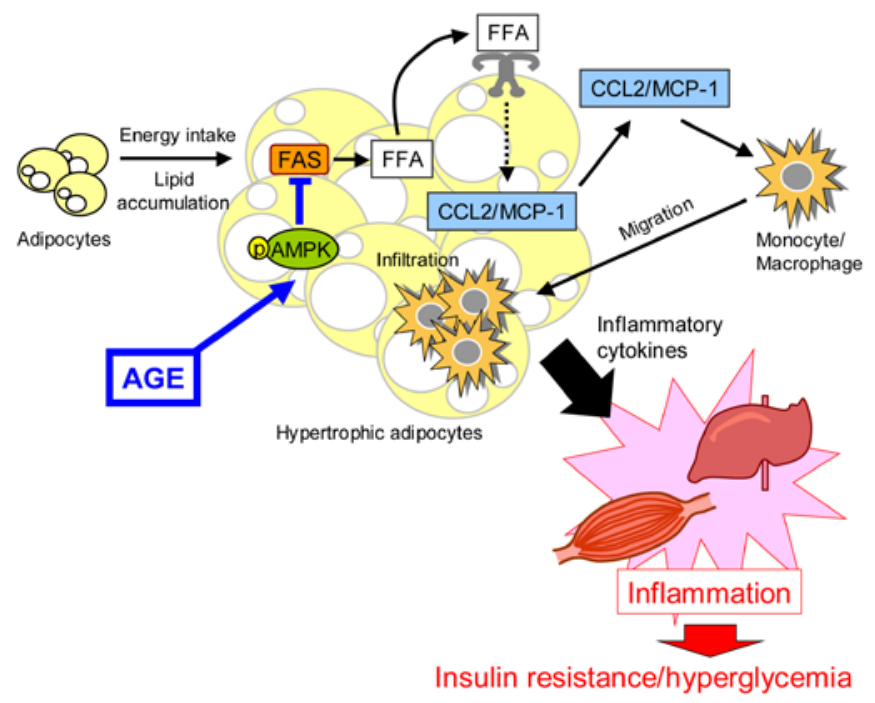

Figure 2. Effect of AGE on hyperglycemia. FFA induces the production of CCL2/MCP-1 via TLR. CCL2/MCP-1 recruits monocytes/macrophages to several tissues. AGE inhibits the production of FFA via the suppression of FAS by AMPK activation, and improves insulin resistance and hyperglycemia by reducing TLR-mediated inflammation in various tissues. AGE, aged garlic extract; AMPK, AMP-activated protein kinase; CCL2, C-C motif chemokine 2; FAS, fatty acid synthase; FFA, free fatty acid; MCP-1, monocyte chemotactic protein-1; TLR, Toll-like receptor.

signaling and the destruction of adipose homeostasis $(57,58)$. Furthermore, monocytes and macrophages infiltrate into adipose tissues and produce inflammatory cytokines, such as TNF- $\alpha$, interleukin (IL)- $1 \beta$ and IL-18 (59-62). AMPK is a key molecule for the prevention and/or amelioration of type 2 diabetes and insulin resistance $(39,63)$. Metformin has been used for the therapy of diabetes through the activation of AMPK by inhibiting mitochondrial complex I of the respiratory chain $(64,65)$. Treatment with metformin has been shown to reduce blood glucose levels, inhibit hepatic gluconeogenesis and improve insulin sensitivity $(66,67)$. In addition, resveratrol has been shown to increase glucose uptake and mitochondrial biogenesis via the activation of AMPK (68-70). Furthermore, berberine has been shown to improve glucose intolerance, reduce body weight, increase the expression of the insulin receptor and LDLR, lower total and LDL cholesterol levels, and reduce triglyceride levels $(71,72)$. Therefore, the activation of AMPK helps ameliorate type 2 diabetes.

AGE has been shown to increase the phosphorylation of AMPK in Tsumura Suzuki Obese Diabetes (TSOD) mice, a mouse model of type 2 diabetes (34). In addition, AGE suppresses the plasma glycated albumin level and improves glucose intolerance in TSOD mice. The level of glycated albumin is proportional to the amount of glucose in plasma as glucose nonenzymatically reacts with the amino group of proteins in plasma (73,74). Blood glucose is taken up via glucose transporter 4 (GLUT4) in skeletal muscle and adipose tissue $(75,76)$. However, the production of GLUT4 is impaired through the inhibition of insulin signaling by pro-inflammatory cytokines in diabetes $(75,76)$. Accordingly, inflammation induces insulin resistance and hyperglycemia. 
Previous studies have suggested that AGE suppresses inflammation in clinical trials and animal studies $(23,28,29,34)$. AGE has been shown to inhibit the expression of Ccl2/Mcp- 1 mRNA in adipose tissue and liver of TSOD mice (34). PRRs, including TLRs trigger the production of inflammatory cytokines and chemokines by recognizing antigens, whereas PRRs have been reported to recognize not only foreign pathogens, but also cellular components (12-14). Saturated free fatty acids (FFA) from adipose tissues have been reported to activate TLR4 and trigger the production of inflammatory cytokines in immune cells $(57,58)$. The level of FFA is regulated by fatty acid synthase (FAS), and AMPK negatively regulates the expression of Fas mRNA by modulating SREBP-1c (45,77). AGE has inhibited the expression of Fas mRNA via the activation of AMPK (34). Therefore, AGE can ameliorate hyperglycemia and insulin resistance by anti-inflammatory effect via the activation of AMPK in TSOD mice (Fig. 2).

\section{Conclusions}

AGE ameliorates atherosclerosis and type 2 diabetes through the suppression of inflammation. In addition, AGE and its components induce the activation of AMPK in several tissues and cells. AMPK plays an important role in regulating the inflammatory response through the inhibition of the TLR signaling pathway. Thus, AMPK is considered as a possible therapeutic target for inflammatory-related diseases. Therefore, AGE, which has a promoting effect on AMPK activation, may prove to be a useful preparation for the prevention and improvement of various diseases associated with chronic inflammation.

\section{Acknowledgements}

The authors would like to thank Dr Takami Oka of Wakunaga Pharmaceutical Co., Ltd. for his helpful advice, encouragement and critical reading of the manuscript.

\section{Funding}

No funding was received.

\section{Availability of data and materials}

Not applicable.

\section{Authors' contributions}

SM, JIS and NM conceived this review. SM, KK and JIS analyzed the relevant literature. SM wrote the manuscript and constructed the figures. JIS, KK and NM critically revised the manuscript. All authors have read and approved the final manuscript.

\section{Ethics approval and consent to participate}

Not applicable.

\section{Patient consent for publication}

Not applicable.

\section{Competing interests}

The authors declare that they have no competing interests.

\section{References}

1. Hardie DG, Ross FA and Hawley SA: AMPK: A nutrient and energy sensor that maintains energy homeostasis. Nat Rev Mol Cell Biol 13: 251-262, 2012

2. Mihaylova MM and Shaw RJ: The AMPK signalling pathway coordinates cell growth, autophagy and metabolism. Nat Cell Biol 13: 1016-1023, 2011.

3. Garcia D and Shaw RJ: AMPK: Mechanisms of Cellular Energy Sensing and Restoration of Metabolic Balance. Mol Cell 66: 789-800, 2017.

4. Bai A, Ma AG, Yong M, Weiss CR, Ma Y, Guan Q, Bernstein CN and Peng Z: AMPK agonist downregulates innate and adaptive immune responses in TNBS-induced murine acute and relapsing colitis. Biochem Pharmacol 80: 1708-1717, 2010.

5. Nath N, Giri S, Prasad R, Salem ML, Singh AK and Singh I: 5-aminoimidazole-4-carboxamide ribonucleoside: A novel immunomodulator with therapeutic efficacy in experimental autoimmune encephalomyelitis. J Immunol 175: 566-574, 2005.

6. Zhao X, Zmijewski JW, Lorne E, Liu G, Park YJ, Tsuruta Y and Abraham E: Activation of AMPK attenuates neutrophil proinflammatory activity and decreases the severity of acute lung injury. Am J Physiol Lung Cell Mol Physiol 295: L497-L504, 2008.

7. Lorenzatti AJ and Servato ML: New evidence on the role of inflammation in CVD risk. Curr Opin Cardiol 34: 418-423, 2019.

8. Geovanini GR and Libby P: Atherosclerosis and inflammation: Overview and updates. Clin Sci (Lond) 132: 1243-1252, 2018.

9. Liu CH, Abrams ND, Carrick DM, Chander P, Dwyer J, Hamlet MRJ, Macchiarini F, PrabhuDas M, Shen GL, Tandon P, et al: Biomarkers of chronic inflammation in disease development and prevention: Challenges and opportunities. Nat Immunol 18: 1175-1180, 2017.

10. Arulselvan P, Fard MT, Tan WS, Gothai S, Fakurazi S, Norhaizan ME and Kumar SS: Role of Antioxidants and Natural Products in Inflammation. Oxid Med Cell Longev 2016: 5276130, 2016.

11. Conti P and Shaik-Dasthagirisaeb Y: Atherosclerosis: A chronic inflammatory disease mediated by mast cells. Cent Eur J Immunol 40: 380-386, 2015.

12. Piccinini AM and Midwood KS: DAMPening inflammation by modulating TLR signalling. Mediators Inflamm 2010: 672395, 2010.

13. Vénéreau E, Ceriotti $\mathrm{C}$ and Bianchi ME: DAMPs from Cell Death to New Life. Front Immunol 6: 422, 2015.

14. Patel S: Danger-Associated Molecular Patterns (DAMPs): The Derivatives and Triggers of Inflammation. Curr Allergy Asthma Rep 18: 63, 2018.

15. Matsutomo T and Kodera Y: Development of an Analytic Method for Sulfur Compounds in Aged Garlic Extract with the Use of a Postcolumn High Performance Liquid Chromatography Method with Sulfur-Specific Detection. J Nutr 146: 450S-455S, 2016.

16. Kodera Y, Ushijima M, Amano H, Suzuki JI and Matsutomo T: Chemical and biological properties of $S$-1-propenyl-l-cysteine in aged garlic extract. Molecules 22: E570, 2017.

17. Nantz MP, Rowe CA, Muller CE, Creasy RA, Stanilka JM and Percival SS: Supplementation with aged garlic extract improves both NK and $\gamma \delta$-T cell function and reduces the severity of cold and flu symptoms: A randomized, double-blind, placebo-controlled nutrition intervention. Clin Nutr 31: 337-344, 2012.

18. Xu C, Mathews AE, Rodrigues C, Eudy BJ, Rowe CA, O'Donoughue A and Percival SS: Aged garlic extract supplementation modifies inflammation and immunity of adults with obesity: A randomized, double-blind, placebo-controlled clinical trial. Clin Nutr ESPEN 24: 148-155, 2018.

19. Suzuki JI, Kodera Y, Miki S, Ushijima M, Takashima M, Matsutomo T and Morihara N: Anti-inflammatory action of cysteine derivative $S$-1-propenylcysteine by inducing MyD88 degradation. Sci Rep 8: 14148, 2018.

20. Kyo E, Uda N, Kasuga S and Itakura Y: Immunomodulatory effects of aged garlic extract. J Nutr 131 (3s): 1075S-1079S, 2001.

21. Morihara N, Ide N and Weiss N: Aged garlic extract inhibits CD36 expression in human macrophages via modulation of the PPARgamma pathway. Phytother Res 24: 602-608, 2010. 
22. Morihara N, Hino A, Yamaguchi T and Suzuki J: Aged garlic extract suppresses the development of atherosclerosis apolipoprotein E-knockout mice. J Nutr 146: 460S-463S, 2016.

23. Morihara N, Hino A, Miki S, Takashima M and Suzuki JI: Aged garlic extract suppresses inflammation in apolipoprotein E-knockout mice. Mol Nutr Food Res 61: 1700308, 2017.

24. Matsumoto S, Nakanishi R, Li D, Alani A, Rezaeian P, Prabhu S, Abraham J, Fahmy MA, Dailing C, Flores F, et al: Aged Garlic Extract Reduces Low Attenuation Plaque in Coronary Arteries of Patients with Metabolic Syndrome in a Prospective Randomized Double-Blind Study. J Nutr 146 427S-432S, 2016.

25. Budoff M: Aged garlic extract retards progression of coronary artery calcification. J Nutr 136 (Suppl): 741S-744S, 2006.

26. Ried K, Frank OR and Stocks NP: Aged garlic extract reduces blood pressure in hypertensives: A dose-response trial. Eur J Clin Nutr 67: 64-70, 2013.

27. Matsutomo T, Ushijima M, Kodera $Y$, Nakamoto $M$, Takashima M, Morihara N and Tamura K: Metabolomic study on the antihypertensive effect of $S$-1-propenylcysteine in spontaneously hypertensive rats using liquid chromatography coupled with quadrupole-Orbitrap mass spectrometry. J Chromatogr B Analyt Technol Biomed Life Sci 1046: 147-155, 2017.

28. Ried K, Travica N and Sali A: The effect of aged garlic extract on blood pressure and other cardiovascular risk factors in uncontrolled hypertensives: The AGE at Heart trial. Integr Blood Press Control 9: 9-21, 2016

29. Ried K, Travica N and Sali A: The Effect of Kyolic Aged Garlic Extract on Gut Microbiota, Inflammation, and Cardiovascular Markers in Hypertensives: The GarGIC Trial. Front Nutr 5: 122, 2018.

30. Hiramatsu K, Tsuneyoshi T, Ogawa T and Morihara N: Aged garlic extract enhances heme oxygenase-1 and glutamate-cysteine ligase modifier subunit expression via the nuclear factor erythroid 2-related factor 2-antioxidant response element signaling pathway in human endothelial cells. Nutr Res 36 : 143-149, 2016.

31. Tsuneyoshi T, Kunimura K and Morihara N: S-1-Propenylcysteine augments $\mathrm{BACH} 1$ degradation and heme oxygenase 1 expression in a nitric oxide-dependent manner in endothelial cells. Nitric Oxide 84: 22-29, 2019.

32. Thomson M, Al-Qattan KK, Js D and Ali M: Anti-diabetic and anti-oxidant potential of aged garlic extract (AGE) in streptozotocin-induced diabetic rats. BMC Complement Altern Med 16: 17, 2016.

33. Ushijima M, Takashima M, Kunimura K, Kodera Y, Morihara N and Tamura K: Effects of $S$-1-propenylcysteine, a sulfur compound in aged garlic extract, on blood pressure and peripheral circulation in spontaneously hypertensive rats. J Pharm Pharmacol 70 559-565, 2018

34. Miki S, Inokuma KI, Takashima M, Nishida M, Sasaki Y, Ushijima M, Suzuki JI and Morihara N: Aged garlic extract suppresses the increase of plasma glycated albumin level and enhances the AMP-activated protein kinase in adipose tissue in TSOD mice. Mol Nutr Food Res 61: 1600797, 2017.

35. Hwang YP, Kim HG, Choi JH, Do MT, Chung YC, Jeong TC and Jeong HG: $S$-allylcysteine attenuates free fatty acid-induced lipogenesis in human HepG2 cells through activation of the AMP-activated protein kinase-dependent pathway. J Nutr Biochem 24: 1469-1478, 2013.

36. Yu L, Di W, Dong X, Li Z, Xue X, Zhang J, Wang Q, Xiao X, Han J, Yang Y, et al: Diallyl trisulfide exerts cardioprotection against myocardial ischemia-reperfusion injury in diabetic state, role of AMPK-mediated AKT/GSK-3 $\beta / \mathrm{HIF}-1 \alpha$ activation. Oncotarget 8: 74791-74805, 2017.

37. Xiao J, Guo R, Fung ML, Liong EC, Chang RC, Ching YP and Tipoe GL: Garlic-Derived $S$-Allylmercaptocysteine Ameliorates Nonalcoholic Fatty Liver Disease in a Rat Model through Inhibition of Apoptosis and Enhancing Autophagy. Evid Based Complement Alternat Med 2013: 642920, 2013.

38. Kim J, Yang G, Kim Y, Kim J and Ha J: AMPK activators: Mechanisms of action and physiological activities. Exp Mol Med 48: e224, 2016.

39. Coughlan KA, Valentine RJ, Ruderman NB and Saha AK: AMPK activation: A therapeutic target for type 2 diabetes? Diabetes Metab Syndr Obes 7: 241-253, 2014.

40. Bäck M, Yurdagul A Jr, Tabas I, Öörni K and Kovanen PT: Inflammation and its resolution in atherosclerosis: Mediators and therapeutic opportunities. Nat Rev Cardiol 16: 389-406, 2019.
41. Salminen A, Hyttinen JM and Kaarniranta K: AMP-activated protein kinase inhibits NF- $x \mathrm{~B}$ signaling and inflammation: Impact on healthspan and lifespan. J Mol Med (Berl) 89: 667-676, 2011.

42. Salminen A and Kaarniranta K: AMP-activated protein kinase (AMPK) controls the aging process via an integrated signaling network. Ageing Res Rev 11: 230-241, 2012.

43. Ou H, Liu C, Feng W, Xiao X, Tang S and Mo Z: Role of AMPK in atherosclerosis via autophagy regulation. Sci China Life Sci 61: 1212-1221, 2018

44. Liu-Bryan R: Inflammation and intracellular metabolism: New targets in OA. Osteoarthritis Cartilage 23: 1835-1842, 2015.

45. Li Y, Xu S, Mihaylova MM, Zheng B, Hou X, Jiang B, Park O, Luo Z, Lefai E, Shyy JY, et al: AMPK phosphorylates and inhibits SREBP activity to attenuate hepatic steatosis and atherosclerosis in diet-induced insulin-resistant mice. Cell Metab 13 376-388, 2011.

46. Doddapattar P, Radović B, Patankar JV, Obrowsky S, Jandl K, Nusshold C, Kolb D, Vujić N, Doshi L, Chandak PG, et al: Xanthohumol ameliorates atherosclerotic plaque formation, hypercholesterolemia, and hepatic steatosis in ApoE-deficient mice. Mol Nutr Food Res 57: 1718-1728, 2013.

47. 47. Wang Q, Zhang M, Liang B, Shirwany N, Zhu Y and Zou $\mathrm{MH}$ : Activation of AMP-activated protein kinase is required for berberine-induced reduction of atherosclerosis in mice: The role of uncoupling protein 2. PLoS One 6: e25436, 2011.

48. Van Gaal LF, Mertens IL and De Block CE: Mechanisms linking obesity with cardiovascular disease. Nature 444: 875-880, 2006.

49. Shoelson SE, Lee J and Goldfine AB: Inflammation and insulin resistance. J Clin Invest 116: 1793-1801, 2006.

50. Rameshrad M, Maleki-Dizaji N, Soraya H, Toutounchi NS, Barzegari A and Garjani A: Effect of A-769662, a direct AMPK activator, on Tlr-4 expression and activity in mice heart tissue. Iran J Basic Med Sci 19: 1308-1317, 2016.

51. Mancini SJ, White AD, Bijland S, Rutherford C, Graham D, Richter EA, Viollet B, Touyz RM, Palmer TM and Salt IP: Activation of AMP-activated protein kinase rapidly suppresses multiple pro-inflammatory pathways in adipocytes including IL-1 receptor-associated kinase-4 phosphorylation. Mol Cell Endocrinol 440: 44-56, 2017.

52. Malekpour-Dehkordi Z, Javadi E, Doosti M, Paknejad M, Nourbakhsh M, Yassa N, Gerayesh-Nejad S and Heshmat R: $S$-Allylcysteine, a garlic compound, increases ABCA1 expression in human THP-1 macrophages. Phytother Res 27: 357-361, 2013.

53. Kemmerer M, Wittig I, Richter F, Brüne B and Namgaladze D AMPK activates LXR $\alpha$ and ABCA1 expression in human macrophages. Int J Biochem Cell Biol 78: 1-9, 2016.

54. Olokoba AB, Obateru OA and Olokoba LB: Type 2 diabetes mellitus: A review of current trends. Oman Med J 27: 269-273, 2012.

55. Kahn SE, Cooper ME and Del Prato S: Pathophysiology and treatment of type 2 diabetes: Perspectives on the past, present, and future. Lancet 383: 1068-1083, 2014.

56. DeFronzo RA, Ferrannini E, Groop L, Henry RR, Herman WH, Holst JJ, Hu FB, Kahn CR, Raz I, Shulman GI, et al: Type 2 diabetes mellitus. Nat Rev Dis Primers 1: 15019, 2015.

57. de Luca $\mathrm{C}$ and Olefsky JM: Inflammation and insulin resistance. FEBS Lett 582: 97-105, 2008

58. Mancuso P: The role of adipokines in chronic inflammation. ImmunoTargets Ther 5: 47-56, 2016

59. McArdle MA, Finucane OM, Connaughton RM, McMorrow AM and Roche HM: Mechanisms of obesity-induced inflammation and insulin resistance: Insights into the emerging role of nutritional strategies. Front Endocrinol (Lausanne) 4: 52, 2013.

60. León-Pedroza JI, González-Tapia LA, del Olmo-Gil E, Castellanos-Rodríguez D, Escobedo G and González-Chávez A: Low-grade systemic inflammation and the development of metabolic diseases: From the molecular evidence to the clinical practice. Cir Cir 83: 543-551, 2015 (In Spanish).

61. Lumeng $C N$ and Saltiel AR: Inflammatory links between obesity and metabolic disease. J Clin Invest 121: 2111-2117, 2011.

62. Rehman K and Akash MS: Mechanisms of inflammatory responses and development of insulin resistance: How are they interlinked? J Biomed Sci 23: 87, 2016.

63. Zhang BB, Zhou G and Li C: AMPK: An emerging drug target for diabetes and the metabolic syndrome. Cell Metab 9: 407-416, 2009.

64. Hardie DG: AMPK: A target for drugs and natural products with effects on both diabetes and cancer. Diabetes 62: 2164-2172, 2013. 
65. Rena G, Hardie DG and Pearson ER: The mechanisms of action of metformin. Diabetologia 60: 1577-1585, 2017.

66. Rojas LB and Gomes MB: Metformin: An old but still the best treatment for type 2 diabetes. Diabetol Metab Syndr 5: 6, 2013.

67. Setter SM, Iltz JL, Thams J and Campbell RK: Metformin hydrochloride in the treatment of type 2 diabetes mellitus: A clinical review with a focus on dual therapy. Clin Ther 25: 2991-3026, 2003.

68. Lagouge M, Argmann C, Gerhart-Hines Z, Meziane H, Lerin C, Daussin F, Messadeq N, Milne J, Lambert P, Elliott P, et al Resveratrol improves mitochondrial function and protects against metabolic disease by activating SIRT1 and PGC-1alpha. Cell 127: 1109-1122, 2006

69. Price NL, Gomes AP, Ling AJ, Duarte FV, Martin-Montalvo A, North BJ, Agarwal B, Ye L, Ramadori G, Teodoro JS, et al: SIRT1 is required for AMPK activation and the beneficial effects of resveratrol on mitochondrial function. Cell Metab 15: 675-690, 2012

70. Zhu X, Wu C, Qiu S, Yuan X and Li L: Effects of resveratrol on glucose control and insulin sensitivity in subjects with type 2 diabetes: Systematic review and meta-analysis. Nutr Metab (Lond) 14: 60, 2017.

71. Liang $\mathrm{Y}, \mathrm{Xu} \mathrm{X}$, Yin $\mathrm{M}$, Zhang $\mathrm{Y}$, Huang L, Chen $\mathrm{R}$ and Ni J: Effects of berberine on blood glucose in patients with type 2 diabetes mellitus: A systematic literature review and a meta-analysis. Endocr J 66: 51-63, 2019.
72. 72. Lee YS, Kim WS, Kim KH, Yoon MJ, Cho HJ, Shen Y, Ye JM, Lee CH, Oh WK, Kim CT, et al: Berberine, a natural plant product, activates AMP-activated protein kinase with beneficial metabolic effects in diabetic and insulin-resistant states. Diabetes 55: 2256-2264, 2006.

73. Welsh KJ, Kirkman MS and Sacks DB: Role of Glycated Proteins in the Diagnosis and Management of Diabetes: Research Gaps and Future Directions. Diabetes Care 39: 1299-1306, 2016.

74. Anguizola J, Matsuda R, Barnaby OS, Hoy KS, Wa C, DeBolt E, Koke $M$ and Hage DS: Review: Glycation of human serum albumin. Clin Chim Acta 425: 64-76, 2013.

75. Leto D and Saltiel AR: Regulation of glucose transport by insulin: Traffic control of GLUT4. Nat Rev Mol Cell Biol 13: 383-396, 2012.

76. Huang S and Czech MP: The GLUT4 glucose transporter. Cell Metab 5: 237-252, 2007.

77. Bijland S, Mancini SJ and Salt IP: Role of AMP-activated protein kinase in adipose tissue metabolism and inflammation. Clin Sci (Lond) 124: 491-507, 2013.

This work is licensed under a Creative Commons Attribution-NonCommercial-NoDerivatives 4.0 International (CC BY-NC-ND 4.0) License. 